\title{
A DEB model for European sea bass (Dicentrarchus labrax): parameterisation and application in aquaculture
}

\author{
Orestis Stavrakidis-Zachou ${ }^{\mathrm{a}, \mathrm{b}}$, Nikos Papandroulakis ${ }^{\mathrm{a}}$, Konstadia Lika ${ }^{\mathrm{b}, *}$ \\ ${ }^{a}$ Institute of Aquaculture, Hellenic Centre for Marine Research, AquaLabs, 71500 Gournes, Heraklion, Greece \\ ${ }^{b}$ Department of Biology, University of Crete, 71003 Heraklion, Greece
}

\begin{abstract}
The framework provided by the Dynamic Energy Budget (DEB) theory allows the quantification of metabolic processes and the associated biological rates that are of interest for aquaculture, such as growth and feeding. The DEB parameters were estimated for farmed European sea bass (Dicentrarchus labrax), a species of major importance for the Mediterranean aquaculture, using zero- and uni-variate literature data and achieving an overall good fit. The obtained parameter set was used to validate the model on sites representatively covering the geographic distribution of the aquaculture activity in Greece via comparison of model predictions to observations. Inter-individual variability of farmed fish was introduced through: 1) an individual initial weight and 2) a factor that acts as an individual-specific multiplier for some of the model parameters and produces scatter in maximum size, and age and size at puberty. Growth of E. sea bass was adequately predicted by the model while feeding tended to be underestimated, particularly during the period following the summer months when warmer temperatures promote high growth rates. The results suggest robustness of the model since it is able to simulate growth and food intake in several independent aquaculture production units, using a common parameter set. The accuracy of growth predictions supports the applicability of the model in variable environmental conditions in the context of climate change. Reconstruction of the feeding history from growth data revealed variations in the scaled functional response $(f)$, i.e., the feeding rate as fraction of maximum possible one of an individual of a given size, throughout the production cycle. However, model simulations with constant $f$ result in reasonably good predictions for growth and feeding in variable environmental conditions. Tendency of the model to underestimate the feeding process revealed both model weaknesses associated with higher temperatures as well as irregularities in the feeding protocols applied at the farm level. Our work demonstrates the capacity and potential of DEB theory for further development of tools that contribute to the assessment and improvement of feeding practices in aquaculture.
\end{abstract}

Keywords: DEB parameter estimation, interval estimates, inter-individual variability,

Dicentrarchus labrax, European sea bass, aquaculture

\section{Introduction}

European sea bass (Dicentrarchus labrax) is a species of high importance in aquaculture. It was the first non-salmonid marine fish to be commercially farmed in Europe and to date,

\footnotetext{
${ }^{*}$ Coresponding author. Fax: +30 2810394408

Email address: lika@uoc.gr (Konstadia Lika) 
it is the most commercially important fish produced in the Mediterranean areas with Greece, Turkey, Spain and Egypt being the biggest producers. Including Turkey, which is currently a leading country in E. sea bass farming, the total production of the species in Europe has increased exponentially in the last decades with production reaching 158,479 tonnes in 2015 (FEAP, 2016). However, to what extent this trend will continue and how changes such as rising temperatures will affect production remains uncertain.

Rising temperatures constitute the principal characteristic of climate change and the potential implications of this trend on fish, fisheries, aquaculture and the dependent communities in the Mediterranean as well as the rest of Europe have been recognized (Brugre, 2015; Hollowed et al., 2013; Peck et al., 2013; Rosa et al., 2012). For E. sea bass in particular, temperature plays an important role in growth, maturation, gonad differentiation, mortality and behaviour. Although some traits such as protein utilization seem to be enhanced by lower temperatures (Peres and Oliva-Teles, 1999), growth, food intake, nitrogen excretion and oxygen consumption rates tend to increase with temperature, reaching maximum values at $26-27^{\circ} \mathrm{C}$ beyond which point they exhibit slight decrease (Person-Le Ruyet et al., 2004). Higher temperatures promote white muscle growth by increasing both the number and size of muscle cells, but the effect differs between life stages, as shown for eleutheroembryos and larvae (Alami-Durante et al., 2006), which indicates that acquiring information for the whole production cycle is critical. Experimental work based on the metabolic activity of E. sea bass at temperatures ranging from 7 to $30^{\circ} \mathrm{C}$, has provided insight regarding the width of the thermal window of the species (Claireaux et al., 2006; Ozolina et al., 2016; Person-Le Ruyet et al., 2004) while it appears that E. sea bass is able to withstand maximum critical temperatures of $33^{\circ} \mathrm{C}$ for short periods (Ozolina et al., 2016) .

It is evident that despite the large volume of literature regarding the species, significant knowledge gaps exist with respect to its response to prolonged exposure to elevated temperatures as well as the thermal effects on growth at both boundaries of the temperature tolerance range. Due to the economic importance of the species it is imperative that the impact of climate change on the future production of the species be assessed. This highlights the necessity for the development of a model that is able to quantify changes in processes that are relevant for the species culture, such as growth and food utilization, under various dietary and environmental regimes throughout the production cycle.

In finfish aquaculture, various modelling approaches have been used to describe growth. Traditionally, empirical models have been used to study aquaculture production due to their simplicity and their ability to link environmental parameters to state variables using relatively small sets of data without representing the underlying biological processes (Lupatsch et al., 2001; Tveters and Tveters, 2010). Classical bioenergetic models have also been extensively used and they are characterized by intermediate complexity while identifying and estimating a small number of key parameters based on data such as growth and respiration (Libralato and Solidoro, 2008; Piedecausa et al., 2010). Typically, "classical" bioenergetic models use one state variable to characterize the state of an individual, they are parameter-rich, they do not capture the full cycle of the individual in a single modeling framework and they require additional assumptions to relate parameters for different species (Nisbet et al., 2012). Dynamic Energy Budget (DEB) theory on the other hand, is a theory that provides the conceptual and quantitative framework to study the whole life cycle of an individual while making explicit use of energy and mass balances (Kooijman, 2010a). Its applicability in studying growth, feeding or the effects of phenomena like climate change lies in its capacity to capture metabolic processes as a function of temperature and food availability in a dynamic way for the entire life cycle 
of the species in question. DEB models have been successfully used in aquaculture to study bivalves (Guyondet et al., 2010; Sará et al., 2012) and fish such as the Atlantic salmon (Salmo salar) (Fore et al., 2016), the bluefin tuna (Thunnus orientalis) (Jusup et al., 2011) the white seabream (Diplodus sargus) and the gilthead seabream (Sparus aurata) (Serpa et al., 2013).

In this study a DEB model for E. sea bass was developed with the aim to mechanistically describe the processes of growth and food consumption for the whole life cycle of the species with focus on the stages pertinent to aquaculture while accounting for temperature changes. Using literature data the DEB parameters were estimated and their uncertainty was assessed. The model provides quantification of metabolic rates that can be used to assess the response of E. sea bass to changing environmental conditions, namely temperature and feeding, in the context of climate change. Furthermore, it introduces a novel method of addressing interindividual variability. Finally, validation of the model using aquaculture production data aimed at increasing the robustness of the generated predictions.

\section{Materials and methods}

\subsection{Model description}

DEB theory is a powerful theoretical framework for modeling the metabolic dynamics of an individual organism through its entire life cycle. Based on physiological rules for the uptake of food by the organism and its use for maintenance, growth and maturation or reproduction, the theory allows for modelling the processes of feeding, digestion, maintenance, maturation, growth, reproduction and aging. A Holling type II functional response relationship is assumed to exist between food density and feeding rate $\left(\dot{p}_{X}\right)$. The scaled functional response $f$, i.e., feeding rate as fraction of maximum possible one of an individual of a given size, is a quantifier of food availability and takes values between 0 (starvation) and 1 (feeding ad libitum). However, due to the asymptotic nature of the functional response, slight changes in $f$ may translate to large changes in food availability at high food levels. Energy from food is extracted by the organism and added to the reserve via the process of assimilation $\left(\dot{p}_{A}\right)$. Subsequent mobilization $\left(\dot{p}_{C}\right)$ of the reserve allows for growth $\left(\dot{p}_{G}\right)$, which is the increase in structural biomass, maintenance $\left(\dot{p}_{S}\right)$, and development or reproduction $\left(\dot{p}_{R}\right)$. A constant fraction $\kappa$ of the mobilized reserve is allocated to somatic functions, which include somatic maintenance and growth, while the remaining $1-\kappa$ fraction is used for development and reproduction, after subtraction of costs related to maturity maintenance $\left(\dot{p}_{J}\right)$.

An individual is described by four state variables: structure $(V)$, energy reserve $(E)$, reproduction buffer $\left(E_{R}\right)$ and maturity $\left(E_{H}\right)$, the latter defined as the cumulative investment to maturation. The state variables, the energy fluxes and the dynamics of the standard DEB model are summarized in Table 1. The DEB parameters are presented in Table 2. For a more comprehensive description of the DEB theory and a full list of the equations and the nomenclature used we refer to (Kooijman, 2010a).

The standard DEB model includes three life stages (embryos, juveniles and adults) and assumes isomorphic growth over all life stages. Isomorphy implies that surface area is proportional to structural volume to the power $2 / 3$. Most species with larvae phase, however, show metabolic acceleration during the early developmental stages, which frequently is followed by morphological metamorphosis (Kooijman, 2014). Metabolic acceleration means that temporarily the isomorphic individual switches to the V1-morphic mode, meaning that the surface area grows proportionally to structural volume. The extended DEB models that include various forms of metabolic acceleration are classified as a-models (Marques et al., 2018a). When acceleration occurs between birth and metamorphosis the model is named "abj" model. Metabolic 
Table 1: Energy fluxes linked to metabolic processes, state variables, and dynamics of the standard DEB. Brackets [] indicate quantities expressed per unit of structural volume and braces \{\} per unit of structural surface area.

Metabolic process

Assimilation

$$
\dot{p}_{A}=\left\{\dot{p}_{A m}\right\} f L^{2}\left(E_{H} \geq E_{H}^{b}\right)
$$

Mobilization

$$
\dot{p}_{C}=E \frac{\dot{v}\left[E_{G}\right] L^{2}+\dot{p}_{S}}{\left[E_{G}\right] L^{3}+\kappa E}
$$

Somatic maintenance

$$
\dot{p}_{S}=\left[\dot{p}_{M}\right] L^{3}+\left\{\dot{p}_{T}\right\} L^{2}
$$

Maturity maintenance

$$
\dot{p}_{J}=\dot{k}_{J} E_{H}
$$

Growth

$\dot{p}_{G}=\kappa \dot{p}_{C}-\dot{p}_{S}$

Maturation/reproduction $\quad \dot{p}_{R}=(1-\kappa) \dot{p}_{C}-\dot{p}_{J}$

\section{State variables}

V

$L$

E

$E_{H}$

$E_{R}$

\section{Dynamics}

$\frac{d}{d t} V=\frac{\dot{p}_{G}}{\left[E_{G}\right]}$

$\frac{d}{d t} E=\dot{p}_{A}-\dot{p}_{C}$

$\frac{d}{d t} E_{H}=\dot{p}_{R}\left(E_{H}<E_{H}^{p}\right)$

$\frac{d}{d t} E_{R}=\kappa_{R} \dot{p}_{R}\left(E_{H} \geq E_{H}^{p}\right)$
Structural body volume

Volumetric structural length: $V^{1 / 3}$

Energy in reserve

Energy investment into maturation

Energy investment to reproduction 
acceleration accommodates the observed change of shape and the empirical observation that length increases approximately exponentially with age during the early juvenile stage for most fish species. The abj-DEB model has been previously used to model the anchovy Engraulis encrasicolus, the zebrafish Danio rerio, the Pacific bluefin tuna, Thunnus orientalis and many Mediterranean Perciformes (Pecquerie et al., 2009; Augustine et al., 2011; Jusup et al., 2011; Lika et al., 2014). The abj-model was also used in this study for the E. sea bass, Dicentrarchus labrax.

In the present study we assume five life stages (embryo, pre-larvae, larvae, juvenile and adult), with stage transitions occurring when the cumulative investment into maturation, $E_{H}$, exceeds certain thresholds: $E_{H}^{h}$ for hatching, $E_{H}^{b}$ for birth, $E_{H}^{j}$ for metamorphosis, and $E_{H}^{p}$ for puberty. The structural volume at this events has values $V_{h}, V_{b}, V_{j}$ and $V_{p}$, respectively. Birth in the DEB context marks the start of exogenous feeding and metamorphosis the completeness of morphological metamorphosis. Puberty is defined as the time when development ceases and allocation to reproduction commences. The stage of interest for aquaculture is the production stage which covers parts of the juvenile and adult stages and relates to the on-growing phase of the species culture. The on-growing phase commences with the transfer of juveniles to marine cages and ends at harvest.

According to abj-model assumptions, between birth and metamorphosis the individual follows the rules for V1-morphy. Since V1-morphy only concerns the relationship between surface area and structural volume, changes in shape affect only the specific maximum assimilation rate, $\left\{\dot{p}_{A m}\right\}$, and the energy conductance, $\dot{v}$, via the acceleration factor $s_{\mathcal{M}}: s_{\mathcal{M}}\left\{\dot{p}_{A m}\right\}$ and $s_{\mathcal{M}} \dot{v}$. The acceleration factor equals one for embryos and pre-larvae, $\left(V / V_{b}\right)^{1 / 3}$ for larvae (early juveniles) and $\left(V_{j} / V_{b}\right)^{1 / 3}$ for juveniles and adults. Consequently, the dynamics (Table 1) will change via the fluxes $\dot{p}_{A}$ and $\dot{p}_{C}$.

A fundamental concept of DEB theory is that all organic compounds, food $(X)$, faeces $(P)$, structure $(V)$ and reserves $(E)$, are mixtures of polymers such as lipids, proteins and carbohydrates and each of them is represented as a "generalized compound" with fixed stoichiometry. The composition of a generalized compound is expressed as the relative abundance of hydrogen $(\mathrm{H})$, oxygen $(\mathrm{O})$ and nitrogen $(\mathrm{N})$ relative to carbon $(\mathrm{C})$. Thus, for example, a molecule of reserve has the formula $\mathrm{CH}_{n_{H E}} \mathrm{O}_{n_{O E}} \mathrm{~N}_{n_{N E}}$, where $n_{* E}$ are the stoichiometric coefficients, e.g. $n_{N E}$ represents the molar $\mathrm{N}: \mathrm{C}$ ratio of reserve. Each generalized compound has specified chemical potential $\left(\mu_{*}\right)$, specific density $\left(d_{*}\right)$, and molecular weight $\left(w_{*}\right)$. Table 2 gives the values of those auxiliary parameters that are relevant to this study.

The abstract state variables of reserves and structure can be linked to commonly measured quantities which in this case are length and mass. Total length, which is a measurable length for a fish, is related to the structural length $(L)$ with the shape factor $\left(\delta_{M}\right)$ given by

$$
L_{w}=L / \delta_{M}
$$

Body mass of an individual has contributions from structure $(V)$, reserve $(E)$ and (for reproducing adults) energy reserve for reproduction $\left(E_{R}\right)$. Mass quantified as wet weight $\left(W_{w}\right)$ is given by

$$
W_{w}=d_{V w} V+\left(E+E_{R}\right) \frac{w_{E w}}{\mu_{E}}
$$

where $d_{V w}$ is the specific density of wet structure $\left(\mathrm{g} / \mathrm{cm}^{3}\right), w_{E w}$ the molecular weight of wet reserve $\left(\mathrm{g} / \mathrm{mol}\right.$ ) and $\mu_{E}$ the chemical potential of reserve $(\mathrm{J} / \mathrm{mol}$ ) (see for details Kooijman (2010b, Comments on section 3.2.1)). The dry- over wet-weight ratio of structure and reserve 
is $\frac{w_{V d}}{w_{V_{w}}}=\frac{d_{V d}}{d_{V_{w}}}$ and $\frac{w_{E d}}{w_{E w}}=\frac{d_{E d}}{d_{E w}}$, respectively. If we assume that $\frac{d_{V d}}{d_{V_{w}}}=\frac{d_{E d}}{d_{E w}}$ (i.e., the water content of reserve equals that of structure) and $d_{V w}=d_{E w}=1 \mathrm{~g} / \mathrm{cm}^{3}$ (i.e., the specific densities of wet structure and reserves equals that of water, which hold approximately), equation (2) becomes

$$
W_{w}=d_{V w} V+\left(E+E_{R}\right) \frac{w_{E d} d_{V w}}{\mu_{E} d_{V d}}
$$

Mass fluxes of organic (food, faeces, reserves and structure) and mineral (e.g., $\mathrm{CO}_{2}$, nitrogenous waste) compounds can be written as weighted sum of three basic fluxes: assimilation $\left(\dot{p}_{A}\right)$, dissipation $\left(\dot{p}_{D}\right)$ and growth $\left(\dot{p}_{G}\right)$ (Kooijman, 2010a, Chapter 4). Dissipation excludes assimilation and somatic growth overheads and amounts to $\dot{p}_{D}=\dot{p}_{S}+\dot{p}_{J}+\left(1-\kappa_{R}\right) \dot{p}_{R}$ for adults or $\dot{p}_{D}=\dot{p}_{S}+\dot{p}_{J}+\dot{p}_{R}$ for the non-reproductive stages. For the non-reproductive stages, the energy invested to maturation is excreted into the environment in the form of heat and metabolites and does not contribute to the total weight.

Specifically, the feeding rate, $\dot{J}_{X}$, in $\mathrm{g} / \mathrm{d}$, is given by

$$
\dot{J}_{X}=\frac{w_{X d}}{\kappa_{X} \mu_{X}} \dot{p}_{A}
$$

where $w_{X d}$ is the molecular weight of dry food $(\mathrm{g} / \mathrm{mol}), \mu_{X}$ the chemical potential of food $(\mathrm{J} / \mathrm{mol})$, and $\kappa_{X}$ the conversion efficiency of food into assimilated energy. Equation (4) gives the feeding rate in dry mass of food. To convert it into wet mass, $\dot{J}_{X}$ must be multiplied by $\frac{d_{X w}}{d_{X d}}$, where $d_{X d}$ is the specific density of dry food $\left(\mathrm{g} / \mathrm{cm}^{3}\right)$ and $d_{X w}$ of wet, which is taken, as above, approximately equal to $1 \mathrm{~g} / \mathrm{cm}^{3}$.

The rate of nitrogenous waste, in the form of ammonia, is given by

$$
\dot{J}_{N_{H}}=\eta_{N A} \dot{p}_{A}+\eta_{N D} \dot{p}_{D}+\eta_{N G} \dot{p}_{G}
$$

where the weight coefficients follow from mass conservation: $\eta_{N A}=\frac{n_{N X}}{\kappa_{X} \mu_{X}}-\frac{n_{N E}}{\mu_{E}}-\frac{n_{N P} \kappa_{P}}{\kappa_{X} \mu_{P}}, \eta_{N D}=\frac{n_{N E}}{\mu_{E}}$ and $\eta_{N G}=\frac{n_{N E}}{\mu_{E}}-\frac{n_{N V} d_{V d}}{\left[E_{G}\right] w_{V d}}$.

Metabolic rates depend on temperature. For a species-specific range of temperatures, the temperature effect is quantified by the Arrhenius relationship (Kooijman, 2010a). For the species-specific Arrhenius temperature $T_{A}$, the rate of a physiological process $\dot{k}$ at temperature $T$ is given by

$$
\dot{k}(T)=\dot{k}_{1} \exp \left(\frac{T_{A}}{T_{1}}-\frac{T_{A}}{T}\right)
$$

where $\dot{k}_{1}$ is the rate at a chosen reference temperature, here $T_{1}=293 \mathrm{~K}$. The reduction of rates at low and high temperatures is modeled based on the idea that metabolic rates are controlled by enzymes that catalyze reactions and that they have inactive configurations outside the temperature tolerance range (Kooijman, 2010a). The rates of enzyme deactivation at the lower $\left(T_{L}\right)$ and upper $\left(T_{H}\right)$ boundaries of the tolerance range are taken to depend on temperature in a way similar to the reaction that is catalyzed by the enzyme, but with different Arrhenius temperatures, $T_{A L}$ and $T_{A H}$, respectively. Therefore, the reduction of physiological rates is quantified by multiplication of the rate with the fraction $s(T) / s\left(T_{1}\right)$ of the enzyme catalyzing the reaction that is in active state at temperature $T$, where

$$
s(T)=\left(1+\exp \left(\frac{T_{A L}}{T}-\frac{T_{A L}}{T_{L}}\right)+\exp \left(\frac{T_{A H}}{T_{H}}-\frac{T_{A H}}{T}\right)\right)^{-1}
$$




\subsection{Data}

Information on the physiology, morphology and life history of the European sea bass was taken both from published literature (Lika et al., 2014; Papandroulakis et al., 2014; PersonLe Ruyet et al., 2004; Zanuy and Carrillo, 1985) and the Hellenic Centre for Marine Research (HCMR). Several observations were incorporated into the parameter estimation including both uni-variate and zero-variate data. Zero-variate data, which comprise of single data points, regarding age, length and wet weight at hatch, birth, metamorphosis, puberty and ultimate size were included, taken from HCMR, Fishbase (http://www.fishbase.org/summary/ Dicentrarchus-labrax/) and published literature (Lika et al., 2014). The uni-variate data consisted of: 1) weight-at-age for the on-growing stage (cage stage) at variant temperatures (Papandroulakis et al., 2014), 2) length- and weight-at-age for the larvae development (Lika et al., 2015), 3) feeding rates as a function of wet weight at variant temperatures (digitized from Zanuy and Carrillo (1985)), 4) reproduction rates as a function of wet weight (Mayer et al., 1990) and 5) Total Ammonia Nitrogen (TAN) excretion rates at various temperatures (Person-Le Ruyet et al., 2004). Larvae and on-growing rearing data from HCMR concerned animals produced via the semi-intensive Mesocosm methodology which is known to promote fast larvae and early juvenile growth rates (Papandroulakis et al., 2014) and the on-growing phase took place in the cage farm of the Institute at Souda Bay (west Crete).

For the validation of the model, data on growth performance of E. sea bass (weight-time and feed consumption) were obtained from various commercial farms covering the 2005-2015 period. Average feeding rates were then calculated as the amount of cumulative feed provided per cage divided by the number of fish and the number of days between weight measurements. Temperature data were also provided by the farms and were given as monthly average Sea Surface Temperatures (SST) for the duration of the on-growing period. Temperatures were recorded with traditional in-situ sensors below the conductive laminar sub-layer, where daily changes are minimal and diurnal fluctuations are considered negligible (Kawai and Wada, 2007). Since temperature effects on growth are of particular interest for aquaculture, we used datasets from farms located in three areas which constitute important centres of aquaculture activity and cover representative parts of the geographical distribution of the industry in the country. In order to preserve farm anonymity the areas were characterized as "East", "West" and "South", comprising of grid cells with central geographic coordinates "38.72N, 25.81E", “39.85N, 20.19E" and "35.70N, 24.69E", respectively, and approximate dimensions of $50 \times 100$ $\mathrm{km}$. Eight data sets were used in total, two from each region and two from commercial farms of unknown geographical origin.

\subsection{Parameter estimation}

The parameter estimation procedure is presented in Marques et al. (2018b) and at the online AmP manual (http://www. debtheory.org/wiki/). Parameters were estimated simultaneously from the aforementioned zero- and uni-variate data sets (1-5), on the basis of the minimisation of the symmetric bounded loss function $F=\sum_{i=1}^{n} \sum_{j=1}^{n_{i}} w_{i j} \frac{\left(d_{i j}-p_{i j}\right)^{2}}{d_{i}^{2}+p_{i}^{2}}$, where $n$ is the number of different data sets, $n_{i}$ is the number of data-points of data set $i, w_{i j}$ 's are weight coefficients, $d_{i j}$ 's are data-points, $p_{i j}$ 's are predicted values, $d_{i}=n_{i}^{-1} \sum_{j=1}^{n_{i}} d_{i j}$ is the mean value for dataset $i$ and $p_{i}=n_{i}^{-1} \sum_{j=1}^{n_{i}} p_{i j}$ is the mean predicted value for dataset $i$. All code, data and results are downloadable from AmP (2018) Dicentrarchus labrax version 20180511, which in combination with the freely downloadable DEBtool at http://www.bio.vu.nl/thb/deb/deblab/, allows repeatability of the computations. 
Lack of information regarding metabolic rates at the very edges of the temperature tolerance range does not allow estimation of all five temperature parameters. However, this information is required to increase the applicability of the model in climate change-related studies. In order to obtain a measure of the reduction of physiological rates at the upper and lower boundaries of the tolerance range, we used pseudo-data to increase the identifiability of the temperature parameters $\left(T_{A}, T_{H}, T_{L}, T_{A L}\right.$ and $\left.T_{A H}\right)$. For the pseudo-data, we assumed near-zero values of the TAN excretion rates (determined as $5 \%$ of the maximum value), at the upper, $T_{H}$, and lower, $T_{L}$, temperatures of the positive rate range known from literature (280-306 K) (Freitas et al., 2007; Ozolina et al., 2016).

Due to lack of detailed information some parameters could not be estimated and, therefore, the values of the generalized animal were used (Kooijman, 2010a; Lika et al., 2011). These values include: the maturity maintenance $\left(\dot{k}_{J}=0.002 \mathrm{~d}^{-1}\right)$, the reproduction efficiency $\left(\kappa_{R}=\right.$ $0.95)$, the defecation efficiency of food to faeces $\left(\kappa_{P}=0.1\right)$, the chemical potential parameters $\left(\mu_{V}=500 \mathrm{~kJ} / \mathrm{mol}, \mu_{E}=550 \mathrm{~kJ} / \mathrm{mol}, \mu_{X}=525 \mathrm{~kJ} / \mathrm{mol}\right.$ and $\left.\mu_{P}=480 \mathrm{~kJ} / \mathrm{mol}\right)$ and the molecular weights $\left(w_{X d}=w_{V d}=w_{E d}=23.9 \mathrm{~g} / \mathrm{mol}\right)$. Moreover, the specific cost for structure, which is given by $\left[E_{G}\right]=\frac{\mu_{V} d_{V d}}{\kappa_{G} w_{V d}}$, was fixed to the value $\left[E_{G}\right]=5230 \mathrm{~J} / \mathrm{cm}^{3}$ by assuming a growth efficiency of $\kappa_{G}=0.8$. This value corresponds to a dry-weight over wet-weight ratio of structure and reserve equal to 0.2 for fish (AmP, 2018), which implies that the specific density of dry structure is $d_{V d}=0.2 \mathrm{~g} / \mathrm{cm}^{3}$, given that $d_{V w}=1 \mathrm{~g} / \mathrm{cm}^{3}$.

The conversion efficiency of food into assimilated energy was also fixed to the value $\kappa_{X}=0.68$ (Lupatsch et al., 2001). Lupatsch et al. (2001) obtained this value using food with composition that satisfies the protein and lipid requirements recommended by FAO for standard feed formulation which is what the majority of farms use.

The overall goodness of fit of the model is quantified with the Mean Relative Error (MRE) and Symmetric Mean Squared Error (SMSE) (Marques et al., 2018a,b). The relative error (RE) of each data set can also be computed. MRE can take values in the interval $[0, \infty)$, while SMSE has values in the interval $[0,1]$. Values of MRE and SMSE close to 0 mean that the model predictions are close to the data.

Uncertainty of the point estimates was assessed by computing the marginal confidence intervals, which may be comprised of more than one interval (confidence sets), using the profile method (Marques et al., 2018b). The profile method is a two-step procedure. In the first step, the profile of the loss function for a parameter is obtained. In the second, which is the calibration step, the level of the loss function that corresponds to uncertainty is computed.

The profile of the loss function, $F$, for the parameter of interest is obtained by fixing the parameter to a range of values around the parameter estimate that corresponds to the global minimum, $F_{\min }$, of the loss function and estimate all the other parameters. Then, the corresponding difference with the minimum of the loss function, $F-F_{\min }$, is plotted as function of the parameter of interest (Figure 1, left).

For the calibration step, 1000 Monte Carlo data-sets were generated by adding centered log-normally distributed scatter, with the same coefficient of variation, to the predictions for each data-point (zero- and uni-variate data). Since there is no information on scatter for each of the data-points, the coefficient of variation was set equal to the mean of the absolute difference between observed and predicted values divided by the predicted value, $c v=\frac{1}{n} \sum_{i=1}^{n}\left(\frac{1}{n_{i}} \sum_{j=1}^{n_{i}} \frac{\left|d_{i j}-p_{i j}\right|}{p_{i j}}\right)$, which for this study equals to 0.2. For each Monte Carlo simulation of the data-sets, we found the (global) minimum of the loss function. The cumulative distribution of the difference $X=F-F^{0}$ of the global minima, $F$, and the value in the absence of scatter, $F^{0}$, is used to find the threshold value for the loss function that corresponds to a 
given confidence level. For example, for a confidence level 0.9 the threshold value of the loss function, $F_{c}$, was obtained from $P\left(X \leq F_{c}\right)=0.9$ or equivalently $S\left(F_{c}\right)=P\left(X>F_{c}\right)=0.1$, where $S$ is the survivor function of the difference $F-F^{0}$ (Figure 1, right). The marginal confidence set for the parameter of interest consists of all values of the parameter for which the loss function is below the threshold value.

\subsection{Model validation}

DEB parameter values are individual-specific and are partly under genetic control. Genetic variation is substantial in cultured populations, even in those originating from a limited number of broodstock individuals (Chistiakov et al., 2005). These genetic differences reflect in phenotypic differences such as the ultimate size, and age and size at puberty, among others. The model developed here introduces inter-individual variability through subdivision of the population into a number of cohorts that differ in parameter values and initial conditions. We assume that the parameter values vary between individuals according to the covariation rules which are applied among different species (Kooijman, 2010a), but within a narrower range. The parameter values, as discussed in (Kooijman, 2010a, Chapter 8), covary inter-specifically in a particular way via the zoom factor, $z$, defined as $z=L_{m} / L_{m}^{\text {ref }}$, where $L_{m}=\kappa\left\{\dot{p}_{A m}\right\} /\left[\dot{p}_{M}\right]$ is the maximum structural length and $L_{m}^{\mathrm{ref}}=1 \mathrm{~cm}$ is a reference maximum structural length. The argument for the covariation of the primary parameters is that only parameters that relate to the physical design of the organism depend on the maximum size of the organism. Including the aging module, only four parameters depend on physical design: $\left\{\dot{p}_{A m}\right\}, E_{H}^{b}, E_{H}^{p}$ and $\ddot{h}_{a}$. Therefore, for two species, the physical design parameters relate to each other as: $\left\{\dot{p}_{A m}^{2}\right\}=z\left\{\dot{p}_{A m}^{1}\right\}$, $E_{H 2}^{b}=z^{3} E_{H 1}^{b}, E_{H 2}^{p}=z^{3} E_{H 1}^{p}$ and $\ddot{h}_{a}^{2}=z \ddot{h}_{a}^{1}$.

To introduce the inter-individual variability we assume a factor $\zeta$, defined as $\zeta=z / z^{0}=$ $L_{m} / L_{m}^{0}$, where $z^{0}$ and $L_{m}^{0}$ are, respectively, the zoom factor and the maximum structural length of the "reference" individual with the estimated parameter values (Table 2). Individuals relate to the "reference" individual as: $\left\{\dot{p}_{A m}\right\}=\zeta\left\{\dot{p}_{A m}^{0}\right\}, E_{H}^{b}=\zeta^{3} E_{H 0}^{b}, E_{H}^{p}=\zeta^{3} E_{H 0}^{p}$ and $\ddot{h}_{a}=\zeta \ddot{h}_{a}^{0}$. Individuals were assigned different values of the zoom factor $z$ sampled randomly from a normal distribution with mean $z^{0}$ and standard deviation $\sigma$ or, equivalently, assigned different values of the factor $\zeta$ sampled from a normal distribution with mean one and standard deviation $\sigma / z^{0}$; for either distribution the coefficient of variation is $c v=\sigma / z^{0}$. Therefore, a single parameter, that acts as an individual-specific multiplier for some of the parameters, produces scatter which can then be calibrated based on observations of inter-individual variability.

Model validation was then performed via comparison of the model predictions to data obtained from the farms. For each data set, the temperature data provided by the farms were used as the forcing variable and population variability was introduced through the factor, $\zeta$, and the initial weight, $W_{0}$, which was also considered to be normally distributed with mean the value given by the first weight measurement. For each data set, 500 Monte Carlo simulations were performed. The values of the coefficient of variation for $\zeta$ and $W_{0}$ were, respectively, $c v=0.05$ and $c v=0.2$ and were based on empirical knowledge of the observed variability at common market and stocking sizes. However, the realism of the variability generated here could not be further evaluated due to the absence of reported variability for the farm data.

To investigate the variability in food intake throughout the production cycle, we used an approach that involves a dynamic reconstruction of the scaled functional response using reverse modeling. Using the weight-time data and temperature data from the different farms we backestimated the functional response, $f$. The estimation procedure is similar to that described in section 2.3. The Nead-Melder simplex method was used to find the minimum of the symmetric 
bounded loss function and estimate values of the functional response at specified knots. Additionally, a filter was applied to prevent $f$ from taking values outside the interval $\left[f_{\min }, 1\right]$, where $f_{\min }$ is the minimum scaled functional response that is required to reach puberty (Lika et al., 2014). A cubic Hermite spline was used in order to interpolate between points. Similar approaches have been used to reconstruct $f$ time series from temperature and growth trajectories (Cardoso et al., 2006; Freitas et al., 2009; Lavaud et al., 2018; Pecquerie et al., 2012; Troost et al., 2010).

\section{Results}

\subsection{DEB parameters}

The parameter estimates for the E. sea bass DEB model are given in Table 2 and the computed $90 \%$ marginal confidence intervals for parameters of interest in Table 3. An illustrative example of the generated profile of the loss function for the zoom factor, $z$, and the survivor function of the loss function for parameter estimates for 1000 Monte-Carlo simulations is presented in Figure 1. This type of confidence intervals can only provide a rough indication of the uncertainty in parameter values. Parameters such as $\dot{v}$ and $z$ exhibited relatively narrow confidence intervals while $\kappa$ showed the widest, suggesting that this parameter is not well identified by the data. In general, the confidence intervals were asymmetric around the point estimates. The relative distance of the point estimate was smaller to the lower limit than to the upper limit of the confidence interval, with the exception of $\kappa$ and $\delta_{M}$. Moreover, while for most parameters the distance between the point estimate and the upper limit was smaller than three times that of the lower limit, for maturity thresholds it exceeded eight times.

The parameter estimation resulted in an acceptable goodness of fit, quantified by the mean relative error (MRE) of 0.1301 and the Symmetric Mean Squared Error (SMSE) of 0.1351, giving an overall good match between predictions and observations (Figure 2 and Table 4). For the growth data pertaining to the early developmental stages (Figure 2, b-c) we assumed $f=1$, meaning ad libitum feeding, since intensive feeding protocols are applied at hatcheries to ensure maximum growth. For the data sets referring to the production stage where feeding is more variable, $f$ was estimated from the data. This resulted in values of $f=0.76, f=0.78$ and $f=0.94$ for Figures $2 \mathrm{~b}, 2 \mathrm{~d}$ and $2 \mathrm{f}$ respectively, which provide a representation of the average feeding conditions throughout the production stage.

The model performed particularly well at predicting the growth pattern of E. sea bass with the predicted growth curves accurately matching the observations (Figure 2, a-c). The model captured growth both quantitatively (relative errors between 0.06 and 0.11 ) and qualitatively since seasonal growth changes due to temperature were accurately depicted. Predictions regarding food intake, reproductive output and ammonia excretion were also fairly accurate (Figure 2, d-f) with the relative errors being $0.14,0.18$ and 0.05 , respectively.

Developmental aspects were also captured well by the model (Table 4). Specifically, the age at metamorphosis, the life span, and the wet weight at puberty were most accurately captured, whereas higher deviations were observed for length at hatch, ultimate wet weight, and age at birth. Life history traits for which data were not available were predicted by the model. Namely, the age at puberty was predicted at 723 days with the length at puberty being estimated at 53.4 $\mathrm{cm}$.

\subsection{Model validation}

The validation of the model was performed by comparing the predictions of the model (wet weight and feeding rate as functions of time) to data obtained from the farms (Figures 3 and 4). 
Table 2: DEB parameter values of E. sea bass, corrected for the reference temperature of $\mathrm{T}=20^{\circ} \mathrm{C}$. Brackets [] indicate quantities expressed per unit of structural volume and braces \{\} per unit of structural surface area.

\begin{tabular}{|c|c|c|c|}
\hline Symbol & Value & Unit & Interpretation \\
\hline \multicolumn{4}{|l|}{ Core parameters } \\
\hline$z$ & 2.45 & - & Zoom factor \\
\hline$\left\{\dot{p}_{A m}\right\}$ & $85.44 / 585.85^{\mathrm{a}, \mathrm{b}}$ & $\mathrm{J} \mathrm{cm}^{-2} \mathrm{~d}^{-1}$ & Specific maximum assimilation rate \\
\hline$\dot{v}$ & $0.041 / 0.282^{\mathrm{a}}$ & $\mathrm{cm} \mathrm{d}^{-1}$ & Energy conductance \\
\hline$\kappa$ & 0.56 & - & Allocation fraction to soma \\
\hline$\kappa_{X}$ & $0.68^{\mathrm{c}}$ & - & Digestion efficiency of food to reserves \\
\hline$\kappa_{P}$ & $0.1^{\mathrm{c}}$ & - & Defecation efficiency of food to faeces \\
\hline$\left[\dot{p}_{M}\right]$ & 19.60 & $\mathrm{~J} \mathrm{~cm}^{-3} \mathrm{~d}^{-1}$ & Volume-specific somatic maintenance rat \\
\hline$\left[E_{G}\right]$ & $5230^{c}$ & $\mathrm{~J} \mathrm{~cm}^{-3}$ & Specific costs for structure \\
\hline$E_{H}^{h}$ & 0.14 & $\mathbf{J}$ & Maturity threshold at hatching \\
\hline$E_{H}^{b}$ & 1.61 & $\mathrm{~J}$ & Maturity threshold at birth \\
\hline$E_{H}^{j}$ & 526.16 & $\mathrm{~J}$ & Maturity threshold at metamorphosis \\
\hline$E_{H}^{p}$ & $2.5110^{6}$ & $\mathrm{~J}$ & Maturity threshold at puberty \\
\hline$\dddot{h}_{a}^{H}$ & $1.5510^{-9}$ & $d^{-2}$ & Weibull aging acceleration \\
\hline \multicolumn{4}{|c|}{ Temperature parameters } \\
\hline$T_{A}$ & 7998 & K & Arrhenius temperature \\
\hline$T_{H}$ & 303 & $\mathrm{~K}$ & Higher bound of the tolerance range \\
\hline$T_{L}$ & 274 & $\mathrm{~K}$ & Lower bound of the tolerance range \\
\hline$T_{A H}$ & 87590 & $\mathrm{~K}$ & $\begin{array}{l}\text { Arrhenius temp for the rate of decrease } \\
\text { at higher boundary }\end{array}$ \\
\hline$T_{A L}$ & 22974 & $\mathrm{~K}$ & $\begin{array}{l}\text { Arrhenius temp for the rate of decrease } \\
\text { at lower boundary }\end{array}$ \\
\hline \multicolumn{4}{|c|}{ Conversion parameters } \\
\hline$\delta_{M}$ & 0.148 & - & Shape coefficient \\
\hline$d_{V d}$ & $0.2^{\mathrm{c}}$ & $\mathrm{g} \mathrm{cm}^{-3}$ & Specific density of dry structure \\
\hline$d_{X d}$ & $0.2 / 0.9^{c}$ & $\mathrm{~g} \mathrm{~cm}^{-3}$ & Specific density of dry fresh/pelleted fooc \\
\hline$w_{X d}, w_{V d}, w_{E d}$ & $23.9^{c}$ & $\mathrm{~g} \mathrm{~mol}^{-1}$ & Molecular weight of dry mass \\
\hline$\mu_{X}, \mu_{V}, \mu_{E}, \mu_{P}$ & $525,500,550,480^{\mathrm{c}}$ & $\mathrm{kJ} \mathrm{mol}^{-1}$ & Chemical potentials \\
\hline$n_{N X}, n_{N V}, n_{N E}, n_{N P}$ & $0.15^{\mathrm{c}}$ & - & Molar N:C ratio of dry mass \\
\hline
\end{tabular}

a Values before/after acceleration

b Derived from: $\left\{\dot{p}_{A m}\right\}=\left(z\left[\dot{p}_{M}\right]\right) /\left(\kappa L_{m}^{r e f}\right)$ with $L_{m}^{r e f}=1$

c Fixed. See text for details.

Table 3: Point estimates (PE) and marginal Confidence Intervals (CI) using the profile method.

\begin{tabular}{lll}
\hline Parameter & PE & CI \\
\hline$\dot{v}$ & 0.041 & $(0.018,0.098)$ \\
$\kappa$ & 0.56 & $(0.04,0.89)$ \\
{$\left[\dot{p}_{M}\right]$} & 19.60 & $(11.52,48.40)$ \\
$E_{H}^{h}$ & 0.14 & $(0.01,4.40)$ \\
$E_{H}^{b}$ & 1.61 & $(0.16,24.99)$ \\
$E_{H}^{j}$ & $5.2610^{2}$ & $\left(0.5510^{2}, 88.8610^{2}\right)$ \\
$E_{H}^{p}$ & $2.5610^{6}$ & $\left(0.3910^{6}, 24.4410^{6}\right)$ \\
$\ddot{h}_{a}$ & $1.5510^{-9}$ & $\left(0.0910^{-9}, 80.2510^{-9}\right)$ \\
$z$ & 2.45 & $(0.98,4.69)$ \\
$\delta_{M}$ & 0.148 & $(0.071,0.198)$ \\
\hline
\end{tabular}



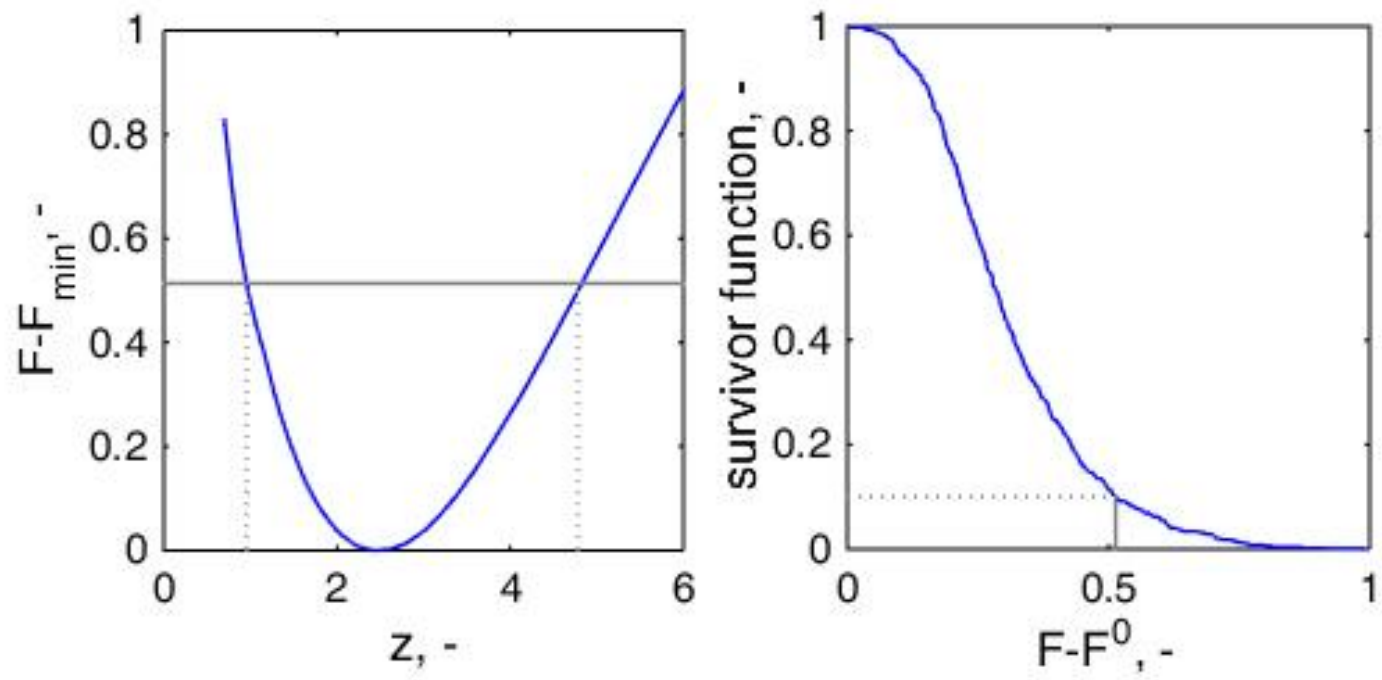

Figure 1: Left: The profile of the loss function for the zoom factor $z$. Right: the survivor function of $F-F^{0}$ (the difference of the minimum of the loss function for 1000 Monte-Carlo simulations minus the value in the absence of scatter). A threshold value (indicated with the solid grey line) for the loss function for a specified uncertainty, here $90 \%$, was obtained from the survivor function plot (see text for details). The marginal confidence interval was obtained by selecting values for the parameter $z$ (left plot) that have loss function values lower than the threshold (horizontal grey line). In this case, the $90 \%$ marginal confidence interval for $z$ was $(0.98,4.69)$ (indicated with the dotted grey lines).

Table 4: Comparison of model predictions with observed age, length and weight (where available) at hatch, birth, metamorphosis and puberty for E. sea bass. Last column gives the relative error (RE) for each data-point.

\begin{tabular}{llllll}
\hline Symbol (unit) & Interpretation & $\mathbf{T}\left({ }^{\mathbf{O}} \mathbf{C}\right)$ & Observations & Predictions & RE \\
\hline$a_{h}(\mathrm{~d})$ & Age at hatch & 15 & 3.6 & 3.92 & 0.09 \\
$a_{h}(\mathrm{~d})$ & Age at hatch & 17 & 2.9 & 3.22 & 0.11 \\
$t_{b}(\mathrm{~d})$ & Time since hatch at birth & 16 & 9 & 8.01 & 0.11 \\
$t_{b}(\mathrm{~d})$ & Time since hatch at birth & 19 & 7 & 6.00 & 0.14 \\
$t_{j}(\mathrm{~d})$ & Time since hatch at metam. & 19 & 70 & 69.88 & $<0.01$ \\
$a_{p}(\mathrm{~d})$ & Age at puberty & 19 & na & 723 & na \\
$a_{m}(\mathrm{~d})$ & Life span & 20 & 5475 & 5472 & $<0.01$ \\
$L_{w}^{h}(\mathrm{~cm})$ & Length at hatch & & 0.35 & 0.22 & 0.37 \\
$L_{w}^{b}(\mathrm{~cm})$ & Length at birth & & 0.55 & 0.50 & 0.10 \\
$L_{w}^{j}(\mathrm{~cm})$ & Length at metam. & & 4 & 3.40 & 0.15 \\
$L_{w}^{p}(\mathrm{~cm})$ & Length at puberty & & na & 53.38 & na \\
$L_{w}^{i}(\mathrm{~cm})$ & Ultimate total length & & 103 & 113.6 & 0.10 \\
$W_{w}^{b}(\mathrm{~g})$ & Weight at birth & & $4310^{-5}$ & $5710^{-5}$ & 0.31 \\
$W_{w}^{p}(\mathrm{~g})$ & Weight at puberty & & 700 & 711 & 0.02 \\
$W_{w}^{i}(\mathrm{~g})$ & Ultimate wet weight & & $1010^{3}$ & 6853 & 0.32 \\
\hline & & & & &
\end{tabular}



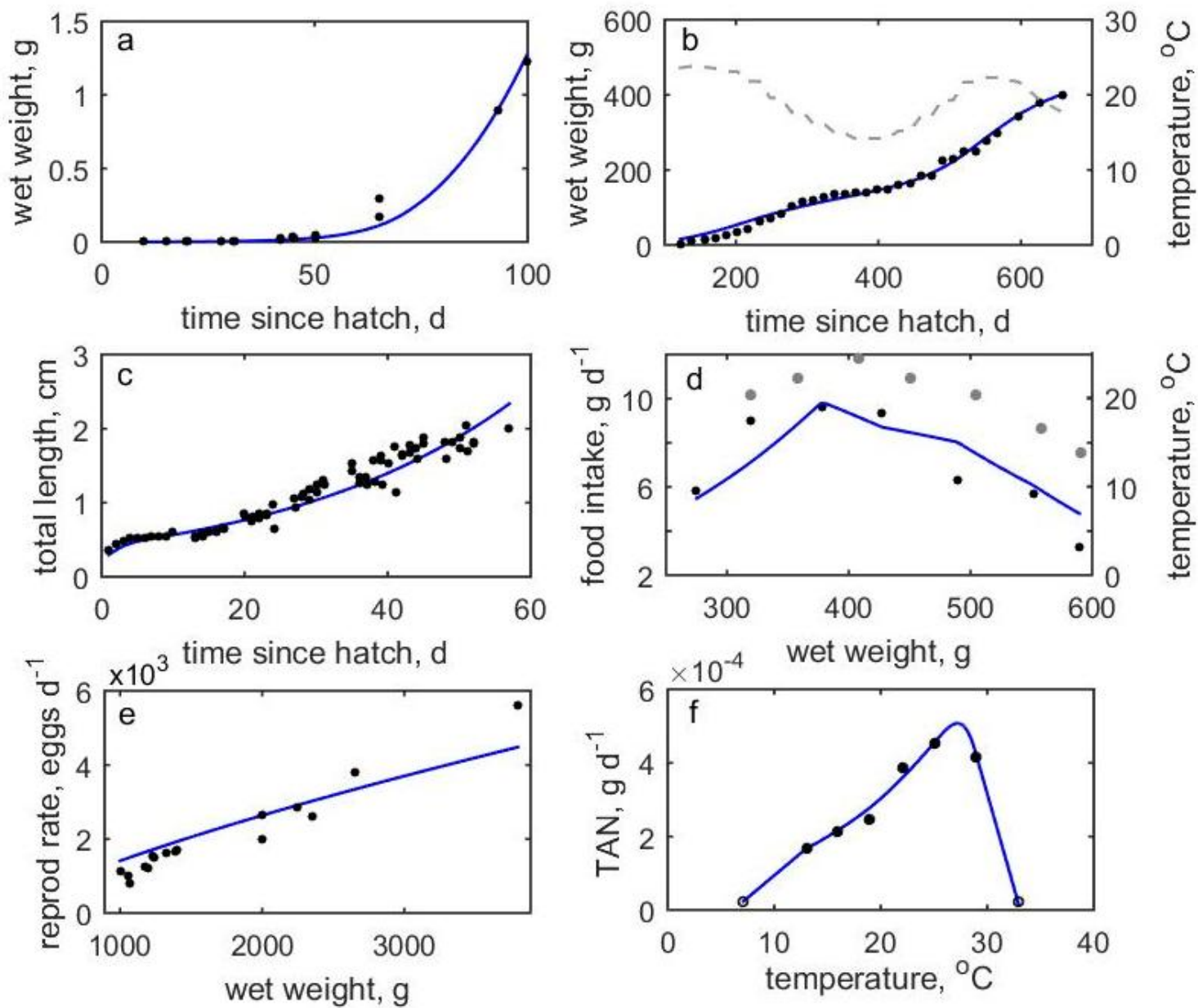

Figure 2: Comparison of model predictions (solid lines) to observations (solid points): a. Weight-at-age at constant temperature. b. Weight-at-age at variable temperature (broken line). c. Length-at-age. d. Food intake as function of weight at variable temperatures (grey points). e. Reproduction rate as function of weight. f. Total Ammonia Nitrogen excretion (TAN) rate as function of temperature (open circles denote pseudo-data). 
For each data set, the temperature data provided by the farms were used as the forcing variable. For all simulations, a constant functional response of $f=0.8$ was assumed. This value was chosen as being about the average of the $f$ values estimated for the univariate data sets used for parameterization. In reality, individual food intake exhibits a degree of daily fluctuation even under controlled conditions. Taking into account that farmed fish are traditionally fed well in order to maximize growth, our results suggest that this assumption can provide a good approximation of the feeding process. Moreover, the food given in the farms is in pelleted form with the dry-to-wet ratio being around 0.9 (Yildiz et al., 2007), so we took $d_{X d}=0.9 \mathrm{~g} / \mathrm{cm}^{3}$.

Overall, the model performed well with considerable accuracy at predicting both growth and food consumption for all data sets (Figures 3 and 4). With respect to predicting the evolution of weight (Figures 3 and 4, a, c, e, g), the model can be characterized by high accuracy. Predictions (solid lines) matched the observations (points) in all eight datasets with high precision and the small deviations from the empirical mean fell within the generated uncertainty (gray shaded areas). The similarity between model predictions and observed growth in production stages indicates that our model features the main mechanisms and effects required to predict the performance in production facilities.

Regarding food consumption (Figures 3 and 4, b, d, f, h), although the model generally captured the pattern of the feeding process, marked deviations were also recorded. In addition, the goodness of fit differed between regions. Predictions of both sets originating from the South region closely matched the observations while for the West region the model consistently underestimated the feeding process. Where differences between model predictions and observations existed, predictions were always lower than the observations, thus showing the tendency of the model to underestimate feeding rates. The highest differences were observed during the period following the summer months when warmer temperatures promote high growth rates. This is depicted on Figure 3-e where high underestimation of the feeding rates during the high summer temperatures of the first year coincides with underestimation of the growth rates, indicating that the model predictive capacity is reduced towards the edges of the temperature tolerance range. However, other datasets, such as the ones from the South region (Figure 3, e-h), suggested that underestimation of feeding is not systematic since both growth and feeding rates are captured well even at temperatures approaching $28^{\circ} \mathrm{C}$. Therefore, the observed deviations may be attributed to both model uncertainty and inconsistencies in the real data.

The reconstruction of the scaled functional response, $f$, was carried out for the three growth data sets presented in Figures $4 \mathrm{a}, 4 \mathrm{e}$ and $4 \mathrm{~g}$. The reconstructed $f$ trajectories are presented in Figure 5. The results suggest that $f$ varies throughout the production cycle with average values $f=0.71$ (Figure 5a), $f=0.76$ (Figure 5b) and $f=0.73$ (Figure 5c). The temporal pattern of the reconstructed $f$ follows that of the growth rate (not shown), where the low values of $f$ correspond to low growth rates in the weight-data.

\section{Discussion}

The parameters of a DEB model for E. sea bass, a species of major importance for the Mediterranean aquaculture, were estimated. The framework provided by the DEB theory allowed the quantification of processes that are of interest for aquaculture such as growth and feeding. Moreover, the novel profile method of Marques et al. (2018b) was applied to assess the uncertainty of the estimated parameters. In general, the confidence intervals were asymmetric around the point estimates. Interestingly, the confidence intervals for all maturity thresholds were the most asymmetric ones, with the relative distance of the point estimate to the lower limit much smaller than to the upper limit. This may indicate strong limitations with respect to 

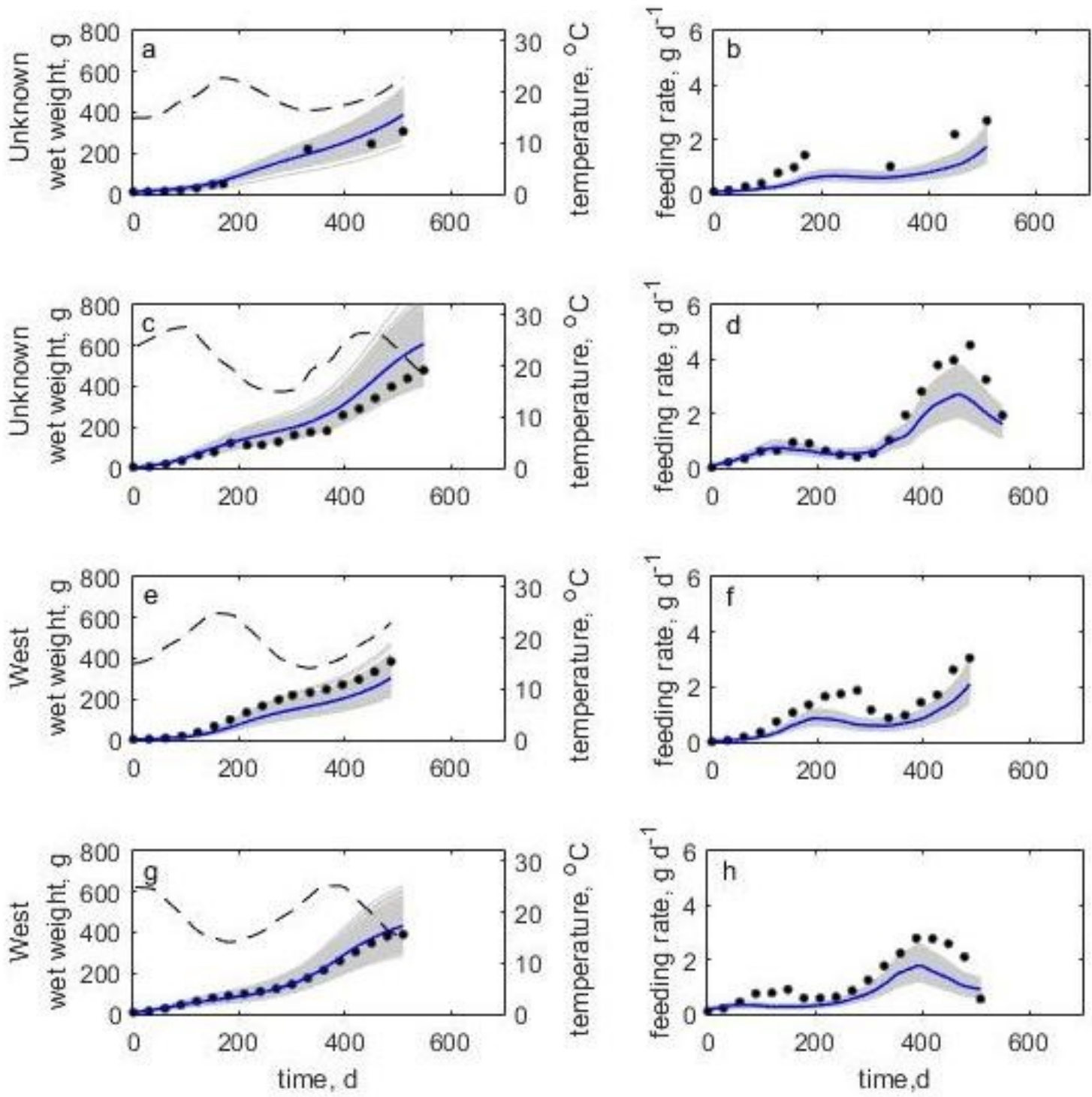

Figure 3: Wet weight $(\mathrm{g})$, feeding rate $(\mathrm{g} / \mathrm{d})$ and temperature $\left({ }^{\circ} \mathrm{C}\right)$ as a function of time $(\mathrm{d})$ for two datasets of unknown origin (a-d) and two from the West (e-h) Region: comparison of model predictions (solid lines) to observations (points). Grey shaded areas indicate model uncertainty (500 Monte Carlo simulations) and broken lines the temperature from each region. For all simulations, $f=0.8$. 

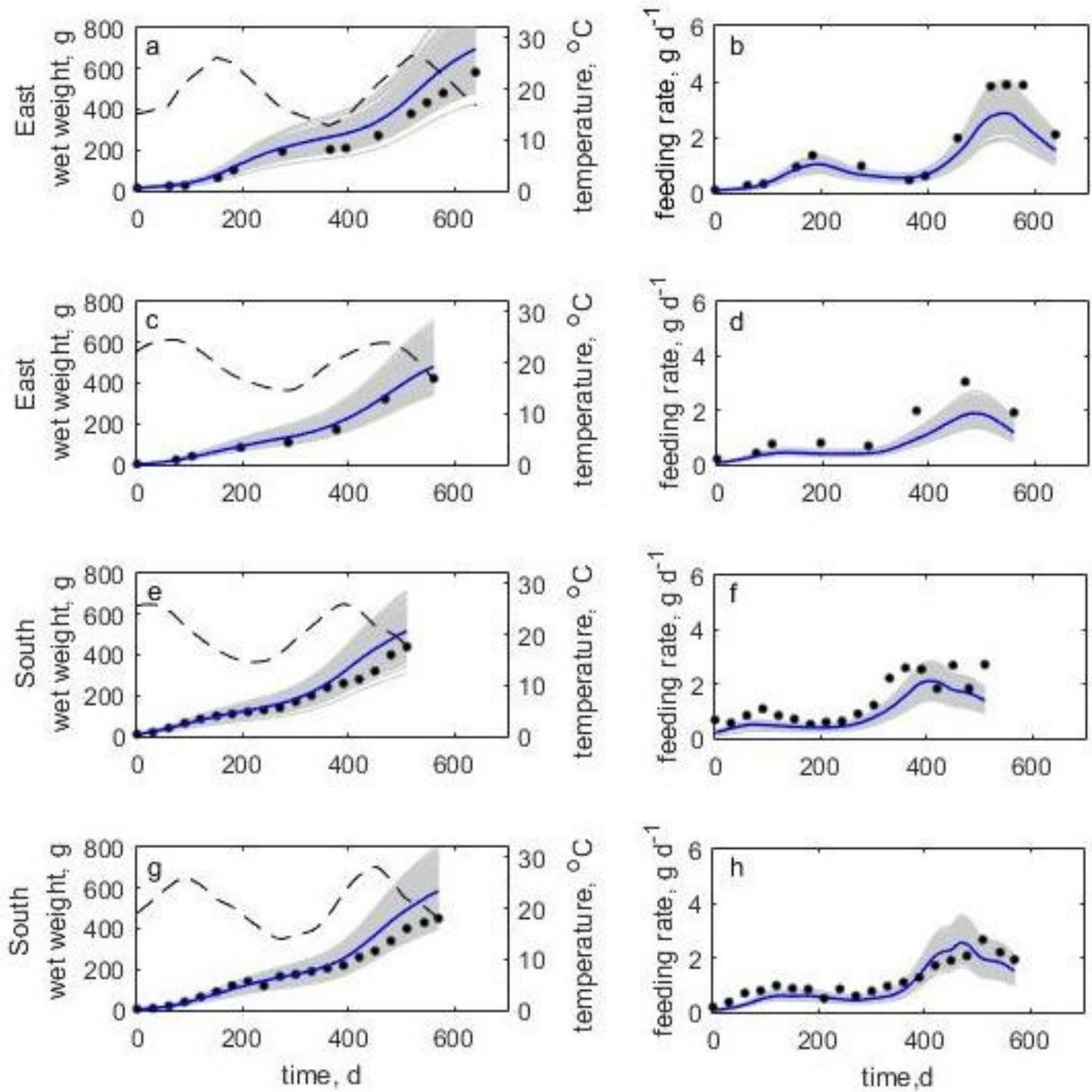

Figure 4: Wet weight $(\mathrm{g})$, feeding rate $(\mathrm{g} / \mathrm{d})$ and temperature $\left({ }^{\circ} \mathrm{C}\right)$ as functions of time $(\mathrm{d})$ for two datasets from the East (a-d) and two from the South (e-h) Region: comparison of model predictions (solid lines) to observations (points). Grey shaded areas indicate model uncertainty (500 Monte Carlo simulations) and broken lines the temperature from each region. For all simulations, $f=0.8$. 

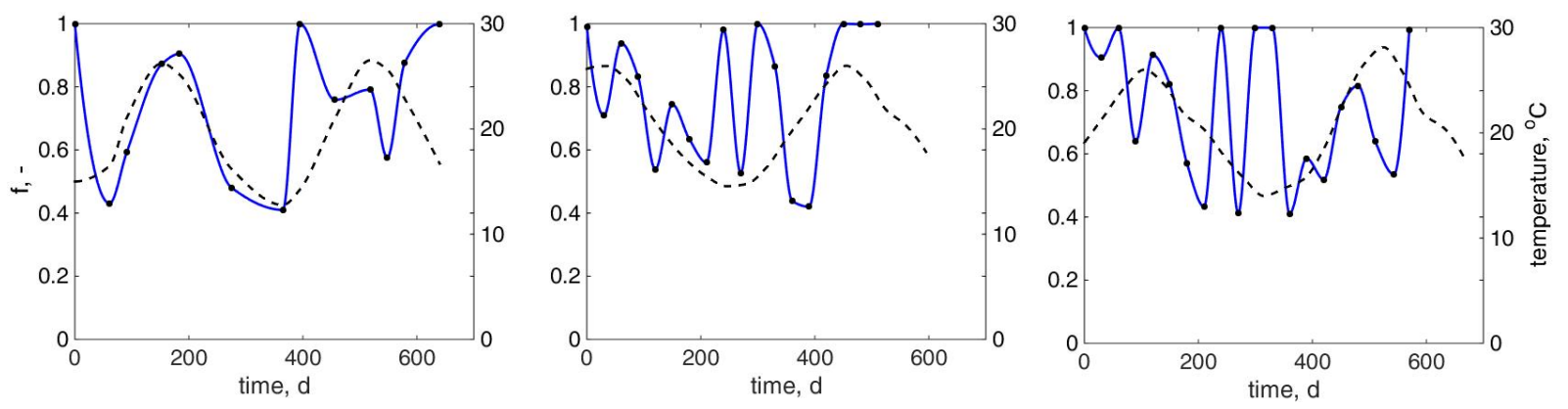

Figure 5: Reconstructed $f$ trajectory from weight-time data from Figure 4a (a), Figure 4e (b) and Figure 4g (c). Solid lines represent the smoothed trajectory through the estimated knot values (points) and broken lines denote temperature.

reducing developmental time for various stages which in turn provides insight into the capacity of aquaculture in selecting for those traits.

For all the regions that were investigated, the single estimated parameter set was capable of reproducing the growth patterns with high accuracy. The growth rates for the eight production sets that were used fell within the range reported for the species for temperate climates (LópezAlbors et al., 2008; Navarro-Martn et al., 2009; Samaras et al., 2017). Temperature between production data-sets differed not only due to the geographic distribution of the farms but also due to the timing of stocking which may differ among or within farms. The good performance of the model for the wide range of temperatures forced in the simulations $\left(14.1-27.9^{\circ} \mathrm{C}\right)$ while capturing the seasonal aspects of growth, highlights the generality and robustness of the developed model. Furthermore, it suggests satisfactory predictive capacity and applicability of the model for studying implications of phenomena like climate change, at least for the temperature range tested here.

Maintaining food availability at maximum (i.e., $f$ close to 1 ) would theoretically allow maximization of growth for farmed species. However, in practice this is neither feasible nor financially viable due to the multi-factorial nature of fish biology and farm economics. In fact, attempting to maintain $f$ close to 1 would likely result in low assimilation efficiency and overfeeding, and thus increased costs and lower overall profitability (Jusup et al., 2014). Moreover, lower feeding levels may be desirable due to the associated benefits in immunology and overall fish health (Mišlov Jelavić et al., 2012).

Varying feeding protocols involving differences in food quality, feeding frequency, duration of feeding, and feeding techniques can alter the efficiency of food conversion (Kousoulaki et al., 2015) and therefore, result in farm-specific $f$ values. Moreover, feeding throughout the production cycle is rarely constant since husbandry practice can modify feeding by including days of starvation on the feeding schedule or prior to handling (vaccination, sampling, harvesting) or restricted rations under adverse rearing conditions (Kousoulaki et al., 2015; Leal et al., 2011). The aforementioned changes in feeding protocols are often implemented at specific stages of the production cycle to accommodate size-dependent physiological changes of the farmed fish. This may explain why discrepancies between observed and predicted feeding rates were more pronounced at larger sizes. However, it is apparent from our simulations that a constant functional response (here $f=0.8$ ) that assumes well-fed fish can yield predictions 
that match growth observations with reasonable accuracy. Therefore, a representative average value for $f$ can provide realistic output to be used for simulating future projections in aquaculture. Other approaches such as trajectory reconstruction can be applied if the research focus is oriented towards describing the temporal variations of the feeding process (Freitas et al., 2009; Jusup et al., 2014; Pecquerie et al., 2012).

Reconstruction of the feeding trajectory for selected farms showed that the average feeding conditions (quantified as $f$ ) of those farms were close to the one we used for validation of the model. Moreover, the reconstruction results suggested that food availability was highly variable throughout the production cycle with oscillating patterns that have also been recorded for other farmed fish (Jusup et al., 2014). Although the time of stocking, and therefore the temperature pattern, differed between the three production sets for which $f$ was reconstructed, no explicit correlation was detected between fluctuations of $f$ and time of stocking or seasonality. This in turn indicates that the observed variability could be interpreted by irregularities in the feeding scheme. Therefore, we conclude that attention should be given in establishing feeding protocols that reduce the aforementioned oscillations in $f$, which would consequently increase the predictability of production.

The boundaries of the temperature tolerance range for the species, and especially the higher bound, constitute areas of scientific uncertainty since limited information is available in the literature regarding the effect of extreme temperatures on metabolic rates (Claireaux et al., 2006; Ozolina et al., 2016). The assumptions we used here to estimate the Arrhenius deactivation rates at the edges of the temperature tolerance range constitute model weaknesses and more experimental work is required in order to decrease model uncertainty. Nevertheless, they provide means of addressing thermal effects on farmed E. sea bass at a wider temperature spectrum, which is pertinent to climate change.

Inevitably, the model is expected to be more sensitive at high temperatures. Although not evident for growth, validation showed tendency of the model to underestimate feeding rates, which was more pronounced in higher temperatures. However, as discussed in section 3.2 this was not consistent. Therefore, despite the model's uncertainty associated with higher temperatures, deviations between predictions and observations could also be attributed to the employed feeding practices.

The model approximates the feeding process and predicts individual daily food intake. However, data originating from farms are not individual-based and rarely exhibit the same level of precision. Feeding in fish farms is a relatively coarse process with the measured and reported quantities relating to the total biomass of feed provided per cage rather than the actually quantities consumed by the fish. Moreover, feeding in cage aquaculture is traditionally determined by observation with indicators such as the decline of fish density at the surface denoting satiation and the end of food supply. This approach however is not accurate and provision occurs in excess with as much as $8 \%$ being lost depending on the species and the feed properties (Garcia-Pineda et al., 2011).

Apart from environmental implications, the financial aspect of overfeeding is the one most important for aquaculture. Feeding represents the main expenditure in finfish aquaculture and can exceed $60 \%$ of the total production cost (Llorens et al., 2017), which renders the improvement of food efficiency a priority for industrial aquaculture. Growth is known to decline at temperatures above the optimal range due to loss of appetite (Pörtner and Knust, 2007). Therefore, overfeeding can be a pronounced event during summer months when feed provision is not timely adjusted to the reduction of growth rates. Such a pattern is consistent with our validation results and suggests that the model developed here can provide means of assessing and 


\section{Acknowledgments}

We are thankful to the Greek aquaculture producers for providing the production data used for validation and P. Anastasiadis for assembling them. We further thank one anonymous referee and L. Pecquerie for their constructive comments and suggestions concerning the paper and S. Augustine for providing the code for the reconstruction of the functional response. Funding was provided through the European Union Horizon 2020 Project ClimeFish 677039.

\section{References}

AmP. Add-my-Pet collection, online database of DEB parameters, implied properties and referenced underlying data. http://www.bio.vu.nl/thb/deb/deblab/add_my_pet/Last accessed: 2018/04/01..

Alami-Durante, H., Rouel, M., Kentouri, M., 2006. New insights into temperature-induced white muscle growth plasticity during Dicentrarchus labrax early life: A developmental and allometric study. Marine Biology 149, $1551-1565$.

Augustine, S., Gagnaire, B., Floriani, M., Adam-Guillermin, C., Kooijman, S. A. L. M., 2011. Developmental energetics of zebrafish, Danio rerio. Comparative Biochemistry and Physiology. Part A, Molecular and Integrative Physiology 159 (3), 275-283.

Brugère, C., 2015. Climate Change Vulnerability in Fisheries and Aquaculture: A synthesis of Six Regional Studies. No. C1104 in FAO Fisheries and Aquaculture Circular. FAO, Rome.

Cardoso, J.F.M.F., Witte, J.I.J., van der Veer, H.W., 2015. Intra- and interspecies comparison of energy flow in bivalve species in Dutch coastal waters by means of the Dynamic Energy Budget (DEB) theory. Journal of Sea Research 56 (2), 182-197. 
Chistiakov, D. A., Hellemans, B., Haley, C. S., Law, A. S., Tsigenopoulos, C. S., Kotoulas, G., Bertotto, D., Libertini, A., Volckaert, F. A. M., 2005. A Microsatellite Linkage Map of the European Sea Bass Dicentrarchus labrax L. Genetics 170 (4), 1821-1826.

Claireaux, G., Couturier, C., Groison, A.-L., 2006. Effect of temperature on maximum swimming speed and cost of transport in juvenile European sea bass (Dicentrarchus labrax). The Journal of Experimental Biology 209 (17), 3420-3428.

FEAP, 2016. European Aquaculture Production Report 2007-2015. Federation of European Aquaculture Producers. Prepared by the FEAP Secretariat.

Føre, M., Alver, M., Alfredsen, J. A., Marafioti, G., Senneset, G., Birkevold, J., Willumsen, F. V., Lange, G., Espmark, s., Terjesen, B. F., 2016. Modelling growth performance and feeding behaviour of Atlantic salmon (Salmo salar L.) in commercial-size aquaculture net pens: Model details and validation through full-scale experiments. Aquaculture 464 (Supplement C), 268-278.

Freitas, V., Campos, J., Fonds, M., Van der Veer, H. W., 2007. Potential impact of temperature change on epibenthic predatorbivalve prey interactions in temperate estuaries. Journal of Thermal Biology 32 (6), 328-340.

Freitas, V., Cardoso, J. F. M. F., Santos, S., Campos, J., Drent, J., Saraiva, S., Witte, J. I., Kooijman, S. A. L. M., Van der Veer, H. W., 2009. Reconstruction of food conditions for Northeast Atlantic bivalve species based on Dynamic Energy Budgets. Journal of Sea Research 62 (2), 75-82.

Garcia-Pineda, M., Sendra, S., Lloret, J., Lloret, G., Jan. 2011. Monitoring and control sensor system for fish feeding in marine fish farms. IET Communications 5 (12),1682-1690.

Guyondet, T., Roy, S., Koutitonsky, V. G., Grant, J., Tita, G., 2010. Integrating multiple spatial scales in the carrying capacity assessment of a coastal ecosystem for bivalve aquaculture. Journal of Sea Research 64, 341359.

Hollowed, A. B., Barange, M., Beamish, R. J., Brander, K., Cochrane, K., Drinkwater, K., Foreman, M. G. G., Hare, J. A., Holt, J., Ito, S.-i., Kim, S., King, J. R., Loeng, H., MacKenzie, B. R., Mueter, F. J., Okey, T. A., Peck, M. A., Radchenko, V. I., Rice, J. C., Schirripa, M. J., Yatsu, A., Yamanaka, Y., 2013. Projected impacts of climate change on marine fish and fisheries. ICES Journal of Marine Science 70 (5), 1023-1037.

Jusup, M., Klanjšček, T., Matsuda, H., 2014. Simple measurements reveal the feeding history, the onset of reproduction, and energy conversion efficiencies in captive bluefin tuna. Journal of Sea Research 94 (Supplement C), 144-155.

Jusup, M., Klanjšček, T., Matsuda, H., Kooijman, S. A. L. M., 2011. A full lifecycle bioenergetic model for bluefin tuna. PLoS ONE 6(7): e21903. https://doi.org/10.1371/journal.pone.0021903

Kawai, Y., Wada, A., 2007. Diurnal Sea Surface Temperature variation and its impact on the atmosphere and ocean: A review. Journal of Oceanography 63, 721-744.

Kooijman, S. A. L. M., 2010a. Dynamic Energy Budget Theory for Metabolic Organisation. Cambridge University Press.

Kooijman, S. A. L. M., 2010b. Dynamic Energy Budget Theory for Metabolic Organisation. Cambridge University Press. Comments at http://www.bio.vu.nl/thb/research/bib/Kooy2010_c.pdf

Kooijman, S. A. L. M., 2014. Metabolic acceleration in animal ontogeny: An evolutionary perspective. Journal of Sea Research 94 (Supplement C), 128-137.

Kooijman, S. A. L. M., Lika, K., 2014. Comparative energetics of the 5 fish classes on the basis of dynamic energy budgets. Journal of Sea Research 94 (Supplement C), 19-28.

Kousoulaki, K., Sther, B.-S., Albrektsen, S., Noble, C., 2015. Review on European sea bass (Dicentrarchus labrax, Linnaeus, 1758) nutrition and feed management: a practical guide for optimizing feed formulation and farming protocols. Aquaculture Nutrition 21 (2), 129-151.

Lavaud R., Jolivet A., Rannou E., Jean F., Strand Ø., Flye-Sainte-Marie J., 2018. What can the shell tell about the scallop? Using growth trajectories along latitudinal and bathymetric gradients to reconstruct physiological history with DEB theory. Journal of Sea Research. doi : 10.1016/j . seares . 2018.04.001

Leal, E., Fernández-Durn, B., Guillot, R., Ríos, D., Cerdá-Reverter, J. M., 2011. Stress-induced effects on feeding behavior and growth performance of the sea bass (Dicentrarchus labrax): a self-feeding approach. Journal of Comparative Physiology 181 (8), 1035-1044.

Li, D., Xu, L., Liu, H., 2017. Detection of uneaten fish food pellets in underwater images for aquaculture. Aquacultural Engineering 78 (Part B), 85-94.

Libralato, S., Solidoro, C., 2008. A bioenergetic growth model for comparing Sparus aurata's feeding experiments. Ecological Modelling 214 (2), 325-337.

Lika K., Augustine S., Pecquerie L., Kooijman S.A.L.M., 2014. The bijection from data to parameter space with the standard DEB model quantifies the supply-demand spectrum. Journal of Theoretical Biology. 354:35-47.

Lika, K., Kearney, M. R., Freitas, V., van der Veer, H. W., van der Meer, J., Wijsman, J. W. M., Pecquerie, L., 
Kooijman, S. A. L. M., 2011. The covariation method for estimating the parameters of the standard Dynamic Energy Budget model I: Philosophy and approach. Journal of Sea Research 66 (4), 270-277.

Lika, K., Kooijman, S. A. L. M., Papandroulakis, N., 2014. Metabolic acceleration in Mediterranean Perciformes. Journal of Sea Research 94, 37-46.

Lika, K., Pavlidis, M., Mitrizakis, N., Samaras, A., Papandroulakis, N., 2015. Do experimental units of different scale affect the biological performance of European sea bass Dicentrarchus labrax larvae?. Journal of Fish Biology 86, 1271-1285.

Llorens, S., Prez-Arjona, I., Soliveres, E., Espinosa, V., 2017. Detection and target strength measurements of uneaten feed pellets with a single beam echosounder. Aquacultural Engineering 78 (Part B), 216-220.

López-Albors, O., Abdel, I., Periago, M. J., Ayala, M. D., Alcázar, A. G., Graciá, C. M., Nathanailides, C., Vázquez, J. M., 2008. Temperature influence on the white muscle growth dynamics of the sea bass Dicentrarchus labrax, L. Flesh quality implications at commercial size. Aquaculture 277 (1), 39-51.

Lupatsch, I., Kissil, G. W., Sklan, D., 2001. Optimization of feeding regimes for European sea bass Dicentrarchus labrax: a factorial approach. Aquaculture 202 (3), 289-302.

Lupatsch, I., Santos, G. A., Schrama, J. W., Verreth, J. A. J., 2010. Effect of stocking density and feeding level on energy expenditure and stress responsiveness in European sea bass Dicentrarchus labrax. Aquaculture 298 (3), $245-250$.

Mayer, I., Shackley, S. E., Witthames, P. R., 1990. Aspects of the reproductive biology of the bass, Dicentrarchus labrax L. Fecundity and pattern of oocyte development. Journal of Fish Physiology 36, 141-148

Marques, G. M, Augustine, S., Lika, K., Pecquerie, L. Kooijman, S. A. L. M., 2018a. The AmP project: Comparing Species on the Basis of Dynamic Energy Budget Parameters. PLOS Computational Biology 14(5): e1006100. https://doi.org/10.1371/journal.pcbi.1006100.

Marques, G. M, Lika, K., Augustine, S., Pecquerie, L. Kooijman, S. A. L. M., 2018b. Fitting Multiple Models to Multiple Data Sets. Submitted to this special issue.

Mišlov Jelavić, K., Stepanowska, K., Grubišič, L., Bubič, T.Š., Katavič, I., 2012. Reduced feeding effects to the blood and muscle chemistry of farmed juvenile bluefin tuna in the Adriatic Sea. Aquaculture Research 43 (2), 317-320.

Navarro-Martín, L., Blázquez, M., Viñas, J., Joly, S., Piferrer, F., 2009. Balancing the effects of rearing at low temperature during early development on sex ratios, growth and maturation in the European sea bass (Dicentrarchus labrax).: Limitations and opportunities for the production of highly female-biased stocks. Aquaculture 296 (3), 347-358.

Nisbet, R. M., Jusup, M., Klanjšček, T., Pecquerie, L., 2012. Integrating dynamic energy budget (DEB) theory with traditional bioenergetic models. The Journal of Experimental Biology 215, 892-902.

Omnes, M.-H., Le Goasduff, J., Le Delliou, H., Le Bayon, N., Quazuguel, P., Robin, J. H., 2017. Effects of dietary tannin on growth, feed utilization and digestibility, and carcass composition in juvenile European sea bass (Dicentrarchus labrax L.). Aquaculture Reports 6 (Supplement C), 21-27.

Ozolina, K., Shiels, H. A., Ollivier, H., Claireaux, G., 2016. Intraspecific individual variation of temperature tolerance associated with oxygen demand in the European sea bass (Dicentrarchus labrax). Conservation Physiology 4 (1).

Papandroulakis, N., Lika, K., Kristiansen, T. S., Oppedal, F., Divanach, P., Pavlidis, M., 2014. Behaviour of European sea bass, Dicentrarchus labrax L., in cages impact of early life rearing conditions and management. Aquaculture Research 45 (9), 1545-1558.

Peck, M. A., Reglero, P., Takahashi, M., Catalán, I. A., 2013. Life cycle ecophysiology of small pelagic fish and climate-driven changes in populations. Progress in Oceanography 116 (Supplement C), 220-245.

Pecquerie, L., Fablet, R., De Pontual, H., Bonhommeau, S., Alunno-bruscia, M., Petitgas, P., A. L. M. Kooijman, S., 2012. Reconstructing individual food and growth histories from biogenic carbonates. Marine Ecology Progress Series 447, 151-164.

Pecquerie, L., Petitgas, P., Kooijman, S. A. L. M., 2009. Modeling fish growth and reproduction in the context of the Dynamic Energy Budget theory to predict environmental impact on anchovy spawning duration. Journal of Sea Research 62 (2), 93-105.

Peres, H., Oliva-Teles, A., 1999. Influence of temperature on protein utilization in juvenile European sea bass (Dicentrarchus labrax). Aquaculture 170 (3), 337-348.

Person-Le Ruyet, J., Mahé, K., Le Bayon, N., Le Delliou, H., 2004. Effects of temperature on growth and metabolism in a Mediterranean population of European sea bass, Dicentrarchus labrax. Aquaculture 237 (1), 269-280.

Piedecausa, M. A., Aguado-Giménez, F., Cerezo-Valverde, J., Hernández-Llorente, M. D., García-García, B., 2010. Simulating the temporal pattern of waste production in farmed gilthead seabream (Sparus aurata), Eu- 
ropean sea bass (Dicentrarchus labrax) and Atlantic bluefin tuna (Thunnus thynnus). Ecological Modelling $221(4), 634-640$.

Pörtner, H. O., Knust, R., 2007. Climate change affects marine fishes through the oxygen limitation of thermal tolerance. Science 315 (5808), 95-97.

Rosa, R., Marques, A., Nunes, M., 2012. Impact of climate change in Mediterranean aquaculture. Reviews in Aquaculture 4, 163-177.

Samaras, A., Pavlidis, M., Lika, K., Theodoridi, A., Papandroulakis, N., 2017. Scale matters: performance of European sea bass, Dicentrarchus labrax, L. (1758), reared in cages of different volumes. Aquaculture Research 48 (3), 990-1005.

Sará, G., Reid, G., Rinaldi, A., Palmeri, V., Troell, M., Kooijman, S. A. L. M., 2012. Growth and reproduction simulation of candidate shellfish species at fish cages in the southern mediterranean: Dynamic Energy Budget (DEB) modelling for integrated multi-trophic aquaculture. Aquaculture 324-325, 259-266.

Serpa, D., Ferreira, P. P., Ferreira, H., da Fonseca, L. C., Dinis, M. T., Duarte, P., 2013. Modelling the growth of white seabream (Diplodus sargus) and gilthead seabream (Sparus aurata) in semi-intensive earth production ponds using the Dynamic Energy Budget approach. Journal of Sea Research 76 (Supplement C), 135-145.

Troost, T.A., Wijsman, J.W.M., Saraiva, S. and Freitas, V., 2010. Modelling shellfish growth with dynamic energy budget models: an application for cockles and mussels in the Oosterschelde (Southwest Netherlands). Philosophical Transactions of the Royal Society B: Biological Sciences 365(1557), 3567-3577.

Tveterȧs, S., Tveterảs, R., 2010. The Global Competition for Wild Fish Resources between Livestock and Aquaculture. Journal of Agricultural Economics 61 (2), 381-397.

Yildiz, M., Sener, E., Timur, M. Effects of variations in feed and seasonal changes on body proximate composition of wild and cultured sea bass (Dicentrarchus labrax L.) . Turkish Journal of Fisheries and Aquatic Sciences 7 , 45-51.

Zanuy, S., Carrillo, M., 1985. Annual cycles of growth, feeding rate, gross conversion efficiency and hematocrit levels of sea bass (Dicentrarchus labrax L.) adapted to two different osmotic media. Aquaculture 44 (1), 11-25. 